\title{
Données actuelles sur les transferts d'atrazine dans l'environnement
}

\author{
M Schiavon ${ }^{1}, \mathrm{JM}$ Portal $^{2}, \mathrm{~F}$ Andreux ${ }^{2}$ \\ ${ }^{1}$ ENSAIA, BP 172-54505 Vandœurre-lès-Nancy Cedex \\ ${ }^{2}$ CNRS-CPB, BP 5, 54501 Vandœuvre-lès-Nancy Cedex, France \\ (Reçu le 29 août 1991; accepté le 17 décembre 1991)
}

\begin{abstract}
Résumé - Les différentes voies de dispersion de l'atrazine dans les sols et l'environnement sont examinées, et les résultats discutés en relation avec les méthodes d'approche respectives. Pour ce qui concerne la volatilisation, les données de la littérature sont limitées et discordantes, car les mesures sont le plus souvent menées dans des conditions très éloignées de la réalité: les valeurs rencontrées pour cette voie de transfert vont, suivant les auteurs, de 0,017 à $60 \%$ de la dose appliquée au sol en 72 h. Les travaux menés en vue d'étudier les transferts par ruissellement ou par lessivage sont nombreux et font appel le plus souvent à des expérimentations réalisées en conditions naturelles - travail au niveau de la parcelle ou semi-naturelles - modèles placés sous conditions naturelles - Ces transferts sont particulièrement observables au cours des 2 mois qui suivent l'application du produit. Leur intensité et leur durée sont essentiellement influencées par le climat, le travail du sol et le couvert végétal. Sur une période d'un an, les pertes en atrazine varient de 0,2 à $2 \%$ de la dose appliquée pour le ruissellement, et de 0,005 à $2 \%$ pour le lessivage. Enfin, il est à noter que l'atrazine est en partie métabolisée dans le sol et que la pollution des nappes phréatiques peut être due davantage à son métabolite chloré, le dé-éthylatrazine, qu'à l'herbicide lui-même.
\end{abstract}

\section{atrazine / volatilisation / ruissellement / lessivage}

Summary - The movement of atrazine in the environment. The different ways in which atrazine is dispersed in soils and in the environment are considered, and the results discussed in relation to the respective methods of study. Literature data regarding volatilization are limited and inconsistent, as measurements are frequently carried out in conditions dissimilar to those in the field: from one author to another, values found range between 0.017 and $60 \%$ of applied doses volatilized in 72 hours. Studies related to the transfers by run-off or leaching are numerous, and frequently use experiments carried out under natural conditions, ie experimental plots, or semi-natural, ie outdoor model experiments. These transfers occur mainly during the 2 first months following application of the chemical. Their intensity and duration are mainly influenced by climate, soil management, and the nature of plant cover. Within a 1 year period, the reported losses in atrazine generally range between 0.2 and $2.0 \%$ of the applied doses for run-off, and between 0.005 and $2.0 \%$ for leaching. It should be noted that atrazine is partly metabolized in soil, and that pollution of phreatic levels can therefore be due more to its chlorinated metabolite (de-ethylatrazine), rather than to the herbicide itself. 


\section{INTRODUCTION}

Les produits phytosanitaires ne présentent un intérêt agronomique que pendant le temps où, appliqués sur le sol ou une culture, ils préservent celle-ci vis-à-vis des adventices, des insectes ravageurs ou des maladies. Lorsque, sous l'action de différents facteurs climatiques, ces produits quittent leur site d'application, ils sont considérés comme des micropolluants, à l'origine de contaminations diffuses du milieu.

En dehors des différents paramètres qui conditionnent la disponibilité de ces molécules par rapport aux processus de transfert (Calvet et Jamet, 1979; Jamet, 1979), ce sont les fortes doses et l'utilisation fréquente qui vont favoriser la contamination du milieu par un produit donné. Parmi les nombreux herbicides homologués en France, l'atrazine occupe une place particulière, dans la mesure où il s'agit de la matière active la plus utilisée pour le désherbage du maïs et dont l'emploi s'étend à la vigne et aux vergers.

Dans les zones agricoles occupées de manière intensive par ces cultures, on constate fréquemment une pollution des rivières (Hörmann et al, 1979; Croll et Fiwem, 1990), des eaux de drainage (Schiavon et Jacquin, 1973; Chevreuil et Chesterikoff, 1979; Boesten, 1987), et des nappes (Wehtje et al, 1983; Rao et al, 1985; Frissel et al, 1987; Isensee et al, 1988, 1990; Schiavon et al, 1990) par l'atrazine, et même par son métabolite majeur, la dé-éthylatrazine (Schiavon et al, 1990). II n'est pas rare, au niveau des captages, que la concentration en résidus s-triaziniques excède la norme européenne autorisée de $0,1 \mu \mathrm{g} . \mathrm{I}^{-1}$ (Babut et al, 1991). Dans le contexte actuel, la présence de résidus d'atrazine dans les eaux superficielles, voire dans les nappes phréatiques, a amené les autorités à réduire les doses appliquées ou même à interdire ce produit dans certains pays de la CEE.

Cette dispersion du pesticide, qui n'est pas spécifique à l'atrazine (Kenimer et al, 1989; Babut et al, 1991), est le résultat d'un transfert du produit par ruissellement et érosion, mais aussi par lessivage, ce qui suppose également la contamination des différents horizons profonds du sol. À ces transferts sous forme soluble, préoccupants par rapport à la nécessité du maintien de la qualité de l'eau, il faut ajouter la possibilité de dispersion du produit par volatilisation, qui constitue une source de pollution de l'air.

Différentes approches sont mises en œuvre pour évaluer l'importance des principales voies de dispersion de l'atrazine. Nous nous proposons de faire le point sur les divers aspects appliqués concernant ces recherches.

\section{LA VOLATILISATION}

Dans la dispersion de l'atrazine appliquée à la surface du sol, la volatilisation est souvent invoquée, bien que ce produit présente une faible tension de vapeur $(0,04 \mathrm{mPa})$. En effet, Kearney et al, (1964), ont montré, à l'aide d'atrazine marquée au ${ }^{14} \mathrm{C}$ déposée à la surface de différents sols, que les pertes étaient de l'ordre de $40 \%$ en $72 \mathrm{~h}$, à une température de $35^{\circ} \mathrm{C}$ et de $60 \%$ à $45^{\circ} \mathrm{C}$. Pour ces auteurs, contrairement à Walker (1972), les pertes paraissent faiblement influencées par la nature du sol, même si l'adsorption tend à diminuer la volatilisation.

L'ampleur du processus est remise en cause par les résultats de Burt (1974). La volatilisation de l'atrazine après application sur 10 sols secs différents, placés à une température de $40^{\circ} \mathrm{C}$ et sous une ventilation de 2 I. $\mathrm{mn}^{-1}$, atteint dans ce cas seulement $11 \%$ de la quantité initiale en $48 \mathrm{~h}$. Glotfelty et Schomburg (1989) indiquent pour leur part une volatilisation de $50 \%$ de la dose d'atrazine appliquée en 500 j $(0,2 \%$ en $48 \mathrm{~h})$. Ces auteurs soulignent le rôle joué d'une manière générale par le travail du sol en conditions naturelles, qui modifie la répartition de l'eau et de la matière organique, le réchauffement et donc la température du sol ainsi que ses caractéristiques par rapport au transport.

D'autres travaux menés en laboratoire confirment l'ampleur du phénomène et soulignent le rôle de la température, de la ventilation et des interactions entre l'atrazine et le support. Toutefois, l'ensemble de ces mesures sont en désaccord avec le caractère persistant observé pour ce produit sous conditions naturelles (Schiavon, 1988a, 1988b). Or, sous ces conditions, l'atrazine peut se trouver dans des situations de température et de ventilation bien supérieures à celles expérimentées dans des conditions de laboratoire.

Des valeurs plus en accord avec le caractère persistant du produit dans le sol sont 
données par Scheunert et al, (1986), même si l'objectif de l'expérimentation n'était pas l'évaluation de la volatilisation de l'atrazine. Dans une expérience menée au laboratoire avec un système fermé d'un volume de 15 I (dessiccateur), balayé par un volume d'air de $10 \mathrm{ml} \cdot \mathrm{mn}^{-1}$, l'atrazine est appliquée sur un sol à $20 \%$ d'humidité et portant des plantules d'orge ou de maïs. Dans ces conditions, les auteurs notent des pertes par volatilisation comprises entre 0,05 et $0,08 \%$ en $7 \mathrm{j}$. Si on considère que sous conditions naturelles, la matière active est au cours du temps progressivement soustraite aux réactions de surface par lessivage et par dégradation (Schiavon, 1980), ces résultats concordent avec ceux obtenus lors de bilans en cases lysimétriques à l'aide d'atrazine marquée au ${ }^{14} \mathrm{C}$. Dans ces cas, on constate, après un an, des déficits de radioactivité compris entre 10 et $30 \%$. Au total, nous pouvons considérer que la volatilisation joue un rôle mineur dans la dissipation de l'atrazine. Ces pertes seraient, au plus, de l'ordre de $3 \%$ de la dose appliquée sur une période d'un an.

La volatilisation contribue à la pollution de l'air, par transfert du produit à l'état de vapeur. Ce n'est pas la seule voie, et il faut signaler, même s'il n'existe pas, à notre connaissance, de valeurs dans la littérature, celle provenant d'une dispersion du produit à l'état adsorbé sur les microparticules de sol entraînées par l'action du vent (Cohen et Pinkerton, 1966).

\section{LES TRANSFERTS LATÉRAUX PAR RUISSELLEMENT À LA SURFACE DES SOLS}

Le transport latéral de l'atrazine sur une grande distance, comme pour tout autre produit présent dans le sol, est soumis à 2 facteurs limitants liés, d'une part, à la topographie (nécessité d'une pente) et, d'autre part, au climat (intervention de précipitations plus ou moins intenses, et donnant lieu ou non à des transports de sédiments). Ce transfert est, par ailleurs, dépendant de la faible solubilité de l'atrazine dans l'eau (33 mg. $\mathrm{l}^{-1}$ à $20^{\circ} \mathrm{C}$ ), de la stabilité de ses interactions avec les constituants du sol et des caractéristiques de celui-ci: texture et stabilité structurale, façons culturales, couvert végétal notamment.
De nombreux travaux ont été réalisés en vue d'évaluer les quantités d'herbicide pouvant être transportées par ruissellement, voire par érosion. Ils ont été menés aussi bien en conditions expérimentales naturelles ou seminaturelles (pluies simulées), que modélisées (précipitations simulées, pente contrôlée, etc). De l'analyse des résultats obtenus, on constate que ces 3 approches sont complémentaires et conduisent à de nombreuses conclusions communes.

Les travaux menés en conditions naturelles présentent l'avantage de fournir des résultats qui intègrent l'action de l'ensemble des facteurs influant sur le transport latéral, mais aussi l'inconvénient de ne représenter que des situations uniques, particulièrement liées aux aléas climatiques. De ce fait, les résultats sont très variables d'une année à l'autre (Hall et al, 1972). Les conditions semi-naturelles, où les précipitations sont simulées, permettent de s'affranchir de la variabilité du climat et de reconstituer, pour un sol et une pente donnés, des situations pluviométriques dont la représentatitivté, par rapport à un climat donné peut être appréciée par référence aux années passées (Triplett et al, 1978). L'expérimentation modélisée permet de contrôler un grand nombre de paramètres (pente, sol, précipitations), mais elle oblige à un essai de reconstitution de la couche de labour; système complexe dont les caractéristiques physiques influent particulièrement sur la dynamique de l'eau et sur le transport.

Compte tenu de ces différentes considérations, tout résultat chiffré n'offre qu'un intérêt relatif, mais l'ensemble des travaux réalisés apporte des informations complètes et très utiles.

Le transport latéral de l'atrazine est essentiellement réalisé sous forme hydrosoluble, et non par les sédiments ( $7-10$ fois moins dans ce dernier cas), même si la concentration de l'herbicide dans ceux-ci est toujours de 2-15 fois plus forte (White et al, 1967; Hall et al, 1972 Hall, 1974; Ritter et al, 1974; Leonard et al, 1979). Par ailleurs Wauchope (1987) note l'absence de relation entre les quantités de sédiments récupérées et d'atrazine exportées. Ceci est en relation avec les forts volumes d'eau mis en jeu et les faibles quantités de sol transportées.

En terme de quantités de produits transportés, Wu (1980) observe, en accord avec White et al (1967), Hall et al (1972), Hall (1974), 
Ritter et al (1974) et Triplett et al (1978), que sous conditions naturelles, la première pluie qui survient, $11 \mathrm{j}$ après le traitement, transporte $55 \%$ des pertes totales, tandis que la deuxième, qui survient $62 \mathrm{j}$ après le traitement, n'en transporte que $32 \%$. D'après Ritter et al, (1974), les transferts sont nuls si les précipitations donnant lieu à un ruissellement se produisent plus de 2 mois après application de l'atrazine. Ceci paraît en contradiction avec les observations de Wu (1980) qui, par ailleurs, enregistre des traces d'herbicide, même en fin de saison. Cette divergence, en partie liée à la limite de détection de la matière active, est propre à la technique de dosage mise en œuvre par chaque auteur.

Globalement, et dans des conditions particulièrement favorables au ruissellement, ces exportations (tableau I) sont généralement comprises entre 0,2 et $2 \%$ et n'excèdent pas $5 \%$ de la dose appliquée. Toutefois, Ritter et al (1974) ont obtenu des exportations atteignant $15,9 \%$. Même si les quantités transférées ne représentent qu'une centaine de g. $\mathrm{h}^{-1} \cdot \mathrm{an}^{-1}$ pour des traitements du sol à la dose préconisée par le fabricant $\left(2,5 \mathrm{~kg} \cdot \mathrm{ha}^{-1}\right)$, on observe que la concentration en herbicide des eaux de ruissellement peut être ponctuellement très élevée et atteindre des valeurs de l'ordre de $8,8 \mathrm{mg} . \mathrm{I}^{-1}$ (Triplett et al, 1978). En outre, Snégaroff (1979) donne, dans le cadre d'une étude concernant les marais charentais, des teneurs très inférieures, comprises entre 0,009 et $0,076 \mathrm{mg} \cdot \mathrm{l}^{-1}$.

Tous les auteurs notent les plus fortes teneurs en atrazine dans les premières eaux de ruissellement obtenues après traitement. D'après Léonard et al, (1979), cette concentration décroît exponentiellement avec le temps et dépend des quantités de résidus présentes à la surface du sol, dans la zone active de ruissellement, lesquelles décrois- sent aussi de la même manière (Frank et Sirons, 1985). Les travaux de White et al (1967) montrent que la concentration en atrazine des eaux de ruissellement diminue au cours d'une phase d'écoulement et pour les écoulements successifs.

Si la quantité, la fréquence et la force des précipitations constituent 3 paramètres importants de la régulation des pertes d'atrazine par ruissellement, il faut également prendre en compte le sol et son mode de travail, le mode d'application, ainsi que le couvert végétal et son stade végétatif (Hall et al, 1983).

Pour Triplet et al, (1978), le travail du sol ne joue qu'un rôle minime, mais Sauer et Daniel (1987), observent des pertes significativement plus faibles pour des sols travaillés suivant des méthodes classiques (labour) que pour ceux pour lesquels on utilise les techniques assurant la protection du sol (tableau II). Les auteurs attribuent ces résultats au rôle joué par les résidus de récolte présents à la surface des sols soumis au travail minimal. Ces résidus de récolte interceptent l'herbicide lors du traitement; celui-ci est ensuite facilement lessivé et se retrouve dans les eaux de ruissellement. Les résultats de Sauer et Daniel (1987) sont toutefois en contradiction avec ceux de Kenimers et al (1989), pour qui la matière organique présente à la surface du sol paraît jouer un rôle protecteur vis-à-vis des transferts par ruissellement (tableau III).

La végétation, par sa densité et son stade de développement, modifie également les mouvements latéraux d'atrazine. En effet, la densité de semis et l'évapo-transpiration dans une certaine mesure, influencent le nombre et le volume des ruissellements, en particulier à partir du stade moyen du cycle de la culture (Hall et al, 1972).

Tableau I. Exportations d'atrazine (matière active) par ruissellement selon différents auteurs.

\begin{tabular}{|c|c|c|c|}
\hline $\begin{array}{c}\text { Dose appliquée } \\
\left(\mathrm{kg} \cdot \mathrm{ha}^{-1}\right)\end{array}$ & $\begin{array}{l}\text { Durée du suivi } \\
\text { (ans) }\end{array}$ & $\begin{array}{l}\text { Pertes (\% de la } \\
\text { dose appliquée) }\end{array}$ & Auteurs \\
\hline 2,3 & 1 & 1 & Wu, 1980 \\
\hline $1,45-4,03$ & 3,5 & $0,2-1,9$ & Léonard et al, 1979 \\
\hline variable & 3 & $0,2-5,7$ & Triplett et al, 1978 \\
\hline 2,2 et 4,5 & 1 & $4,9-5,3$ & Hall, 1974 \\
\hline 3,4 & 2 & $2,5-5,4$ & Ritter et al, 1974 \\
\hline $0,6-9,9$ & 1 & $1,73-2,98$ & Hall et al, 1972 \\
\hline
\end{tabular}


Tableau II. Pertes en atrazine (matière active) dans les eaux de ruissellement pour différents types de travail du sol et époques (d'après Sauer et Daniel, 1987).

\begin{tabular}{lcccc}
\hline & \multicolumn{4}{c}{ Pertes $\left(g\right.$. ha $^{-1}$ ) } \\
\cline { 2 - 5 } \multicolumn{1}{c}{ Travail du sol } & mi-juin & mi-juillet & début juin & fin juin \\
\cline { 2 - 5 } Conventionnel & 5,7 & 2,4 & 117,0 & 8,8 \\
Chisel & 5,5 & 1,9 & 82,4 & 7,8 \\
Billons & 7,8 & 1,4 & 239,0 & 10,3 \\
Non travail & 8,1 & 2,0 & 202,0 & 9,7 \\
\hline
\end{tabular}

Tableau III. Transferts latéraux d'atrazine (matière active) mesurés $24 \mathrm{~h}$ après traitement, sur des miniparcelles traitées à la dose de $2,24 \mathrm{~kg} \cdot \mathrm{ha}^{-1}$. Effets du travail du sol et de la matière organique à sa surface. (D'après Kenimers et al, 1989)

\begin{tabular}{|c|c|c|c|c|c|c|c|}
\hline \multirow[b]{2}{*}{$\begin{array}{l}\text { Pente } \\
(\%)\end{array}$} & \multirow[b]{2}{*}{$\begin{array}{l}\text { Travail } \\
\text { du sol* }\end{array}$} & \multirow[b]{2}{*}{$\begin{array}{c}\text { Matière } \\
\text { organique } \\
\left({\left.\mathrm{kg} . h \mathrm{~h}^{-1}\right)}^{2}\right.\end{array}$} & \multirow[b]{2}{*}{$\begin{array}{c}\text { Ruissellement } \\
(\mathrm{mm})\end{array}$} & \multirow[b]{2}{*}{$\begin{array}{l}\text { Sédiments } \\
(\mathrm{kg})\end{array}$} & \multicolumn{3}{|c|}{ Exportations d'atrazine (g/ha) } \\
\hline & & & & & soluble & adsorbée & total \\
\hline 8,9 & $\mathrm{C}$ & 0 & 3,30 & 16,45 & 58,40 & 0,18 & 58,58 \\
\hline 15,0 & $\mathrm{C}$ & 0 & 3,78 & 23,02 & 68,03 & 0,26 & 88,29 \\
\hline 15,1 & $\mathrm{C}$ & 750 & 3,83 & 7,79 & 37,01 & 0,03 & 37,04 \\
\hline 9,1 & NT & 750 & 0,48 & 0,01 & 0,42 & 0,00 & 0,42 \\
\hline 8,5 & NT & 0 & 0,45 & 0,49 & 6,20 & 0,01 & 6,20 \\
\hline
\end{tabular}

* C: Travail conventionnel, NT: Non-travail

\section{Conclusion}

$\mathrm{Au}$ total, les pertes par ruissellement et érosion sont gouvernées principalement par le volume et l'intensité des précipitations qui interviennent à un moment proche du traitement. Elles sont également dépendantes de la dose, de la formulation, du mode d'application, de la persistance et de la rémanence de la matière active, du type de culture et de sa densité, de la topographie, des caractéristiques physiques du sol (stabilité structurale, humidité au moment des nouvelles précipitations), et des techniques culturales.

\section{LE LESSIVAGE}

L'étude du mouvement vertical de l'atrazine a fait l'objet de nombreux travaux, menés aussi bien en conditions de laboratoire que de plein champ. Nous ne rapporterons ici qu'un nom- bre limité de résultats obtenus en plein champ, soit au niveau de la parcelle, soit à l'aide de cases lysimétriques.

Dans l'étude du mouvement vertical de l'herbicide, les premiers travaux ont porté sur la connaissance de la progression du produit vers les couches profondes du sol, en relation avec une préoccupation d'ordre agronomique, liée à l'évaluation de la persistance et à la quantification des résidus présents dans la couche de labour lors de la mise en place d'une culture de remplacement (Horowitz, 1969; Hall et Hartwig, 1978). Les études orientées vers des préoccupations d'ordre écologique apparaissent sensiblement à la même époque, avec des recherches concernant les iransferts dans le sol au-delà de la couche de labour et sous forme libre, vers les eaux de drainage (Schiavon et Jacquin, 1973). À noter également que ces transferts peuvent s'observer après un temps de résidence de plusieurs mois de l'atrazine dans le sol. Outre la matière active, ce sont alors des produits de dégradation qui sont également 
entraînés dans des proportions variables: dééthylatrazine, dé-isopropyl-atrazine, diaminoatrazine, hydroxyatrazine (Muir et Baker, 1978, Schiavon, 1988a, b).

\section{Étude au niveau de la parcelle}

\section{Mouvement dans le sol}

Même si Brejda et al, (1988) considèrent qu'il n'y a pas de lessivage de l'atrazine au-delà de $15 \mathrm{~cm}$ de profondeur, de nombreux auteurs l'ont détectée à des profondeurs très variables, mais plus importantes. Ceci est lié, encore une fois, aux performances des techniques analytiques et au moment du dosage par rapport à la date de traitement. Ainsi, Southwick et al (1990) observent la présence d'atrazine dans les 30 premiers $\mathrm{cm}$ du sol, pendant une période de l'ordre de $174 \mathrm{j}$ après un traitement à la dose de $1,63 \mathrm{~kg} \cdot \mathrm{ha}^{-1}$ et des précipitations cumulées atteignant $922 \mathrm{~mm}$. Des observations sensiblement identiques sont effectuées par Schiavon (1980). Muir et Baker (1978) dosent pour leur part l'atrazine jusqu'à une profondeur de $40 \mathrm{~cm}$ un an après le traitement, mais dans leur cas, la matière active est minoritaire par rapport à l'hydroxyatrazine, le dé-éthylatrazine et le déisopropylatrazine. La présence de métabolites est par ailleurs signalée par Sirons et al
(1973). De même, Hall et Hartwig (1978, 1990) détectent l'herbicide à 60,90 et $122 \mathrm{~cm}$ de profondeur (tableau IV). La profondeur à laquelle les résidus sont détectés dépend de la dose de traitement, de la date à laquelle est faite la mesure par rapport au moment d'application et des précipitations qui sont intervenues. Huang et Frink (1989) ont montré pour leur part, la présence d'atrazine jusqu'à une profondeur de $2,30 \mathrm{~m}$ pour des sols traités à la dose de $1 \mathrm{~kg} \mathrm{ha}^{-1}$, au cours des 2 années précédant le dosage. Les auteurs notent un profil de distribution du produit dans le sol, lié à celui de la distribution en carbone.

L'évaluation des quantités présentes dans les niveaux du sol situés en dessous de la couche de labour est particulièrement importante, dans la mesure où le produit est alors peu susceptible d'une dégradation, et constitue ainsi une réserve qui sera progressivement transférée vers la nappe phréatique. Dans leurs conditions de travail, Huang et Frink (1989) ont évalué cette réserve de matière active à $1,45 \mathrm{~kg} \cdot \mathrm{ha}^{-1}$.

\section{Transfert sous forme libre}

Si la nappe phréatique peut être alimentée en composés s-triaziniques par le biais de résidus qui progressent à la suite de désorptions et adsorptions successives, elle peut égale-

Tableau IV. Distribution de l'atrazine (matière active) dans les différents niveaux du sol en fonction de la dose de traitement et de la date du dosage (d'après Hall et Hartwig, 1978).

\begin{tabular}{|c|c|c|c|c|c|c|c|c|c|}
\hline \multirow{3}{*}{$\begin{array}{l}\text { Atrazine } \\
\text { appliquée } \\
\left(\mathrm{kg}_{\mathrm{ha}} \mathrm{h}^{-1}\right)\end{array}$} & \multicolumn{7}{|c|}{ Atrazine $\left(m g . \mathrm{kg}^{-1}\right)$} & & \multirow[t]{3}{*}{$\%$ dosé } \\
\hline & \multicolumn{8}{|c|}{ Niveaux du sol $(\mathrm{cm})$} & \\
\hline & $0-15$ & $15-30$ & $30-46$ & $46-61$ & $61-76$ & $76-91$ & $91-107$ & $107-122$ & \\
\hline
\end{tabular}

Dosage 2 mois après le traitement (212,8 $\mathrm{mm}$ de précipitations)

$\begin{array}{llllllllll}1,1 & 0,15 & - & 0,01 & - & - & - & - & - & 32 \\ 2,2 & 0,38 & 0,01 & 0,03 & 0,02 & 0,03 & - & - & - & 47 \\ 4,5 & 1,12 & 0,02 & 0,07 & 0,03 & 0,06 & - & - & - & 65,0 \\ 9,0 & 0,75 & 0,02 & 0,16 & 0,02 & 0,10 & 0,07 & 0,10 & 0,06 & 36,5\end{array}$

Dosage 4 mois après le traitement $(478,3 \mathrm{~mm}$ de précipitations)

\begin{tabular}{|c|c|c|c|c|c|c|c|c|c|}
\hline 1,1 & - & - & - & - & - & - & - & - & 0,0 \\
\hline 2,2 & 0,01 & - & 0,04 & - & - & - & - & - & 5,0 \\
\hline 4,5 & 0,20 & 0,16 & 0,06 & - & - & - & - & - & 21,0 \\
\hline 9,0 & 0,66 & 0,08 & 0,15 & 0,08 & - & - & - & - & 24,3 \\
\hline
\end{tabular}


ment être alimentée par des produits dissous dans l'eau libre du sol. La mesure de la concentration en atrazine présente dans les eaux de drainage donne de ce fait des informations sur les quantités de produits pouvant parvenir dans la nappe sous forme libre et d'une manière directe, en l'absence d'un réseau d'évacuation des excédents d'eau.

Les concentrations en atrazine enregistrées dans les eaux de drainage sont très variables. Elles fluctuent entre 0,006 et $0,29 \mathrm{mg}^{-\mathrm{I}^{-1}}$ (Schiavon et Jacquin, 1973; Muir et Baker, 1976; Von Stryk et Bolton, 1977; Southwick et al, 1990). Ces concentrations diminuent avec le temps qui sépare le traitement de la phase de drainage, mais on observe, au cours d'une même phase, que les premières eaux sont les plus riches en résidus (Schiavon et Jacquin, 1973).

$\mathrm{Ce}$ transfert vertical est dépendant de l'adsorption, de la demi-vie de l'atrazine (24 mois), des caractéristiques physico-chimiques du sol et de la distribution des précipitations. Tout aussi variables sont les valeurs concernant l'évaluation des quantités exportées, exprimées en pourcentage par rapport aux doses appliquées (tableau V). Cette variabilité est étroitement liée au type de sol, à la topographie, à la nature et à la distribution des précipitations au cours de l'année.

\section{Études à l'aide de cases lysimétriques ou de colonnes de sol}

L'utilisation de cases lysimétriques placées sous conditions naturelles pour l'étude du mouvement vertical des pesticides est relativement courante (Jarczyk, 1983; 1987; Führ,
1985; Brumhard et al, 1987; Albanis et al, 1988; Bowman, 1989). Ce mode d'investigation présente au moins 2 avantages: d'une part il donne des résultats qui intègrent, comme au niveau de la parcelle, les effets des conditions climatiques naturelles et éventuellement ceux dus à la végétation et aux pratiques culturales. D'autre part, il permet l'utilisation de molécules marquées au ${ }^{14} \mathrm{C}$ et donc une analyse plus fine dans l'identification et la quantification des produits issus de la dégradation de la molécule mère ( $\mathrm{Ku}$ biak et al, 1988).

Concernant l'atrazine, peu d'études ont fait appel à ce modèle. On peut cependant rappeler les travaux d'Albanis et al (1988) à l'aide de lysimètres de $1 \mathrm{~m}$ de côté, ceux de Bowman (1989) et de Schiavon (1980, 1988a, b) avec des colonnes de sol à structure non perturbée.

\section{Mouvement dans le sol}

L'utilisation de l'atrazine marquée au ${ }^{14} \mathrm{C}$ a permis de montrer sans ambiguïté que le produit appliqué à une dose normale $\left(1,6 \mathrm{~kg} \cdot \mathrm{ha}^{-1}\right.$ de matière active), se distribue sur l'ensemble de la colonne et parvient, en un an, à des profondeurs de $60 \mathrm{~cm}(0,82 \%$ de la dose appliquée). Toutefois, plus de la moitié des résidus reste dans le niveau de surface (tableau VI). Cette distribution est étroitement dépendante des précipitations et de leur date d'intervention par rapport au moment du traitement (Bowman, 1989).

Tableau V. Atrazine exportée sous forme libre (matière active). Valeurs obtenues par différents auteurs.

\begin{tabular}{ccccl}
\hline $\begin{array}{c}\text { Dose } \\
\left(\mathrm{kg} \cdot \mathrm{ha}^{-1}\right)\end{array}$ & $\begin{array}{c}\text { Nature } \\
\text { des sols }\end{array}$ & \% exporté & $\begin{array}{c}\text { précipitations } \\
(\mathrm{mm})\end{array}$ & \multicolumn{1}{c}{ Auteurs } \\
\hline 1,25 & argileux & $1,94(1972)$ & 578 & Schiavon et Jacquin (1973) \\
1,25 & argileux & $0,85(1973)$ & 564 & Schiavon et Jacquin (1973) \\
1,25 & limoneux & $0,54(1972)$ & 578 & Schiavon et Jacquin (1973) \\
1,25 & limoneux & $0,005(1973)$ & 564 & Schiavon et Jacquin (1973) \\
1,4 & - & 0,15 & - & Muir et Baker (1976) \\
1,20 & argileux & $0,25-0,80$ & - & Von Stryk et Bolton (1977) \\
$1,2-2,5$ & limon & $0,004-0,01$ & - & Chevreuil et Chesterikoff (1979) \\
1,4 & argileux & 2 & - & Snégaroff (1979) \\
- & limon fin & 0,038 & 530 & Southwick et al (1990) \\
\hline
\end{tabular}


Tableau VI. Distribution de la radioactivité s-triazinique et des résidus extractibles après une période d'un an, à la suite d'un traitement à la dose de $1,6 \mathrm{~kg} \cdot \mathrm{ha}^{-1}$ d'atrazine marquée au ${ }^{14} \mathrm{C}$ sur le cycle $s$-triazinique (d'après Schiavon, 1988a).

\begin{tabular}{cccc}
\hline Niveau & $\begin{array}{c}\text { Distribution de } \\
\text { la radioactivité } \\
\left(\%^{b}\right)\end{array}$ & $\begin{array}{c}\text { Résidus extractibles } \\
(\%)\end{array}$ & $\begin{array}{c}\text { Radioactivité } \\
\text { extractible (\%/niveau) }\end{array}$ \\
\hline $0-6$ & 36,50 & 12,80 & 35,18 \\
$6-12$ & 14,47 & 5,81 & 40,21 \\
$12-18$ & 7,91 & 3,38 & 42,69 \\
$18-24$ & 6,09 & 2,69 & 44,11 \\
$24-30$ & 3,52 & 1,50 & 42,77 \\
$30-36$ & 2,30 & 0,99 & 42,74 \\
$36-42$ & 1,84 & 0,81 & 43,44 \\
$42-48$ & 1,09 & 0,55 & 50,60 \\
$48-54$ & 0,82 & 0,44 & 54,35 \\
& & & \\
Total & 74,54 & 28,97 & \\
Déficit & 25,46 & 71,03 & \\
\hline
\end{tabular}

a: Radioactivité évaluée par combustion; b: Pourcentage établi suivant le rapport : $\frac{\text { radioactivité mesurée }}{\text { matioactité }}$ a: Radioactivité évaluée par combustion; b: Pourcentage établi suivant le rapport : $\frac{\text { radioactivité mesurèe }}{\text { radivité appliquée - radioactivité lessivée }}$

\section{Transfert sous forme libre}

Pour ce qui concerne le transport sous forme libre, les valeurs figurant au tableau VII montrent que, sous les conditions climatiques données (année 1981-1982, avec $850 \mathrm{~mm}$ de précipitations), $5,62 \%$ de la radioactivité appliquée ont été entraînés par les eaux de percolation sous forme de différents composés s-triaziniques où la dé-éthylatrazine était majoritaire (Schiavon, 1988a).

Dans cette radioactivité lessivée, l'atrazine ne représente qu'environ $10 \%$ (fig 1 ), soit $0,6 \%$ d'atrazine transférée vers la nappe sous forme inchangée. Cette valeur mérite d'être particulièrement soulignée, parce qu'elle représente sensiblement le pourcentage d'atrazine exporté par les eaux de drainage de ce même sol en 1972, et qu'elle montre bien, chose peu connue, que dans la pollution des nappes phréatiques, l'atrazine est minoritaire par rapport à ses métabolites. De plus, cette valeur est très proche de celles obtenues par Albanis et al (1988) avec des cases lysimétriques et différents types de sol, à savoir, après $1324 \mathrm{~mm}$ de précipitations, 0,54, 0,66 et $0,47 \%$ de la dose appliquée sont lessivés, respectivement pour un sol argileux, limoneux et limono-sableux.

Tableau VII. Radioactivité s-triazinique exportée par l'eau de percolation de colonnes de sol traitées à l'atrazine marquée au ${ }^{14} \mathrm{C}$ (d'après Schiavon, 1988a).

\begin{tabular}{|c|c|c|c|c|c|c|c|}
\hline \multirow[t]{2}{*}{ Date des analyses } & \multicolumn{3}{|c|}{1981} & \multicolumn{3}{|c|}{1982} & \multirow[b]{2}{*}{ Total } \\
\hline & 24 sept & 15 oct & 14 déc & 30 jan & 02 mai & 29 juin & \\
\hline $\begin{array}{l}\text { Volumes d'eau } \\
\text { collectés (ml) }\end{array}$ & 464 & 386,5 & 980 & 725 & 522,5 & 197,5 & 3275,5 \\
\hline $\begin{array}{l}\text { Quantités } \\
\text { lessivées }(\mu \mathrm{g})\end{array}$ & 1,72 & 2,74 & 10,44 & 19,82 & 23,97 & 6,59 & 65,28 \\
\hline$\frac{\text { Quantité lessivée }}{\text { Quantité appliquée }} \times 100$ & 0,15 & 0,23 & 0,90 & 1,71 & 2,07 & 0,56 & 5,62 \\
\hline
\end{tabular}




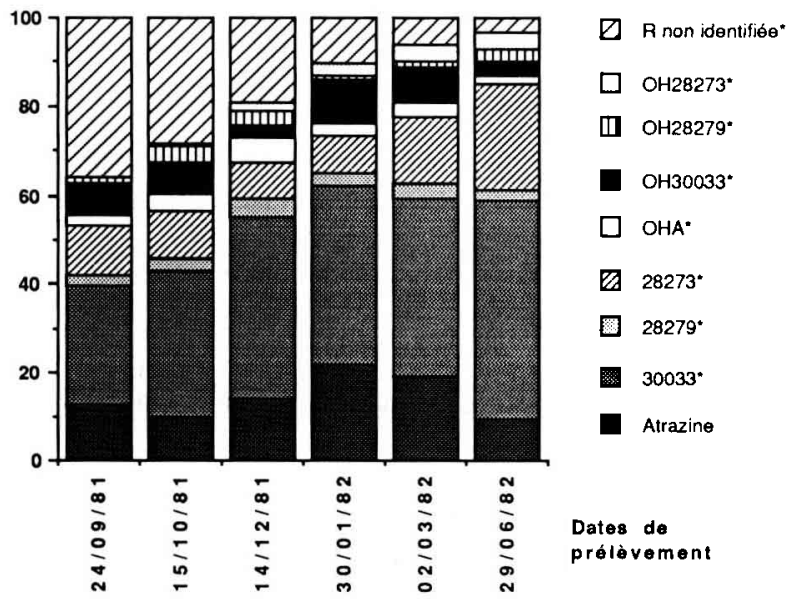

Fig 1. Composition des eaux de percolation de colonnes de sol traitées avec de l'atrazine marquée au ${ }^{14} \mathrm{C}$ sur le noyau $s$-triazinique en fonction des dates de prélèvement et exprimée en pourcentage de la radioactivité présente dans chaque échantillon (d'après Schiavon, 1988a).

* 30033: dé-éthylatrazine, 28279: dé-isopropylatrazine, 28273: dé-éthyl, dé-isopropylatrazine, OHA: hydroxyatrazine, $\mathrm{OH} 30033$ : 2-hydroxy, dé-éthylatrazine, $\mathrm{OH} 28279$ : 2hydroxy, dé-isopropylatrazine, OH28273: 2-hydroxy, dé-éthyl, dé-isopropylatrazine, $R^{*}$ : radioactivité non identifiée

\section{Conclusion}

L'utilisation d'une molécule marquée, qui permet de suivre en simultané le transfert de la matière active et ses produits de dégradation, met en évidence l'effort analytique qui doit être réalisé pour évaluer au niveau de la pratique courante, la pollution réelle du sol et des nappes. S'agissant de l'atrazine, on voit l'effort qui doit être réalisé pour quantifier les métabolites chlorés et surtout hydroxylés.

\section{CONCLUSION}

Les différentes voies qui contribuent à la dispersion de l'atrazine dans l'environnement sous forme inchangée (volatilisation, ruissellement, lessivage), représentent au plus, pour une période d'un an, $10 \%$ de la dose appliquée au sol. Même si l'on ne considère simplement que le lessivage $(\approx 3 \%)$, cela représente des quantités importantes, qui lors d'événements pluvieux proches de la date de traitement conduisent à des concentrations en résidus dans l'eau supérieures à $0,1 \mu \mathrm{g} . \mathrm{I}^{-1}$.
De plus, au cours du temps, par dégradation abiotique ou biologique, prennent naissance un nombre important de métabolites, plus difficiles à doser, et qui sont également soumis à un transfert vers la profondeur du sol et/ou la nappe phréatique.

L'utilisation de la molécule d'atrazine marquée au ${ }^{14} \mathrm{C}$ sur le noyau $s$-triazinique montre que la principale voie de disparition de la matière active dans le sol est constituée par la formation de résidus liés (non extractibles) (Schiavon et al, 1978). Il subsiste le risque que ceux-ci soient ultérieurement libérés d'une manière très diffuse et contribuent secondairement à la pollution de l'environnement. Pour répondre au souci d'une maîtrise de la qualité de l'eau destinée à la consommation, un effort particulier doit être porté sur une quantification précise des transferts de surface et du lessivage tant de la matière active que de ses métabolites.

\section{RÉFÉRENCES}

Albanis TA, Pomonis PJ, Sdoukos AT (1988) Movement of methyl parathion, lindane and atrazine through lysimeters in field conditions. Toxicol Environ Chem 17, 35-45

Babut $M$, Marchand $F$, Schiavon $M$, Thévenot $X$, Français $T$ (1991) Contamination des eaux souterraines par des pesticides et contrôle sanitaire: approche méthodologique à partir de quelques captages lorrains. In: La micropollution organique des eaux et son traitement Coll Assoc Pharm Fr Hydrol, 28 et 29 mars 1991 (sous presse)

Brejda JJ, Shea PJ, Moser LE, Waller SS (1988) Atrazine dissipation and off-plot movement in a Nebraska sandhill subirrigated meadow. J Range Manage 41, 416-420

Boesten JJTI (1987) Leaching of herbicides to ground water: a review of important factors and of available measurements. Br Crop Prot Conf Weeds, 559-568

Bowman BT (1989) Mobility and persistence of the herbicides atrazine, metolachlor and terbuthylazine in plainfield sand determined using field lysimeters. Environ Toxicol Chem 8, 485-491

Brumhard B, Führ F, Mittelstaedt W (1987) Leaching behaviour of aged pesticides: standardized soil column experiments with ${ }^{14} \mathrm{C}$-metamitrone and ${ }^{14} \mathrm{C}$-methabenzothiazuron $\mathrm{Br}$ Crop Prot Conf - Weeds. 585-592 
Burt GW (1974) Volatility of atrazine from plant, soil, and glass surfaces. J Environ Qual 3, 114117

Calvet $R$, Jamet $P$ (1979) Données générales sur le transport des pesticides dans les sols. Trav Soc Pharm Montp 39, 275-288

Chevreuil M, Chesterikoff A (1979) Étude du transfert des pesticides sur un bassin versant de la Brie. Modalités et essai de bilan. CR Séances Acad Agric Fr 65, 835-854

Cohen JM, Pinkerton C (1966) Widespread translocation of pesticides by air transport and rainout. Adv Chem Series 60, 163-176

Croll BT, Fiwem Ph D (1990) Pesticides in surface and underground waters. In: Symp pesticides in soil and water res 11-12 avril 1990 Londres, 1 15

Frank R, Sirons GJ (1985) Dissipation of atrazine residues from soils. Bull Environ Contam Toxicol 34, 541-548

Frisel P, Milde G, Stock R, Ahlsdorf B (1987) Methods and results of an investigation on the impact of agricultural pesticide application on ground-water in northern parts of the Federal Republic of Germany. In: UNO-EEC Sem Protec Soil Aquifers Against Non-point Source Pollution Water/Sem.14/R. Madrid 5-9 octobre 1987, 15, 1-14

Führ F (1985) Application of ${ }^{14} \mathrm{C}$-labeled herbicides in lysimeter studies. Weed Sci 33, 11-17

Glotfelty DE, Schomburg CJ (1989) Volatilisation of pesticides from soil. In: Reactions and movement of organic chemicals in soils Soil. Sci Soc Am Am Soc Agron SSSA Spec Publ 22, 181-207

Hall JK (1974) Erosional losses of s-triazine herbicides. J. Environ Qual 3, 174-180

Hall JK, Hartwig NL (1978) Atrazine mobility in two soils under conventional tillage. J Environ Qual 7, 63-68

Hall JK, Hartwig NL (1990) Triazine herbicide fate in a no-tillage corn (Zea mays L)-crownvetch (Coronilla varia L) "living mulch" system. Agric Ecosyst Environ 30, 281-293

Hall JK, Pawlus M, Higgins ER (1972) Losses of atrazine in runoff water and soil sediment. $J$ Environ Qual 1, 172-176

Hall JK, Hartwig NL, Hoffman LD (1983) Application mode and alternate cropping effects on atrazine losses from a hillside. J Environ Qual $12,336-340$

Horowitz M (1969) Evaluation of herbicide persistence in soil. Weed Res 9, 314-321

Hörmann WD, Tournayre JC, Egli H (1979) Triazine herbicide residues in Central European streams. Pest Monitor J 13, 128-131
Huang LQ, Frink CR (1989) Distribution of atrazine, simazine, alachlor, and metolachlor in soil profils in Connecticut. Bull Environ Contam Toxicol 43, 159-164

Isensee AR, Helling CS, Gish TJ, Kearney PC, Coffman CB, Zhuang W (1988) Groundwater residues of atrazine, alachlor and cyanazine under no-tillage practices. Chemosphere 17 , 165-174

Isensee AR, Nash RG, Helling CS (1990) Effect of conventional vs no-tillage on pesticide leaching to shallow groundwater. J Environ Qual 19 434-440

Jamet P (1979) Le comportement des produits agropharmaceutiques dans le sol. Phytiatr Phytopharm Rev Fr Méd Pharm Vég 28, 87-122

Jarczyk HJ (1983) Investigations on the leaching behaviour of herbicides under natural agronomic conditions of lysimeter-monoliths. Aspects Appl Biol 4, 441-448

Jarczyk HJ (1987) Studies on the leaching characteristics of crop protection chemicals in a monolith lysimeter installation. Pflanzenschutz-Nachr Bayer 40, 49-77

Kearney PC, Sheets TJ, Smith JM (1964) Volatility of seven s-triazines. Weeds 12, 83-87

Kenimers AL, Mostaghimi S, Dillaha TA, Shanholtz VO (1989) Pesticide losses in erosion and runoff simulator. Am Soc Agric Engin 32, 127-136

Kubiak R, Führ F, Mittelstaedt W, Hansper M, Steffens $W$ (1988) Transferability of lysimeter results to actual field situations. Weed Sci 36 , 514-518

Leonard RA, Langdale GW, Fleming WG (1979) Herbicide runoff from upland piedmont watersheds-data and implications for modeling pesticide transport. J Environ Qual 8, 223-229

Muir DC, Baker BE (1976) Detection of triazine herbicides and their degradation products in tiledrain water from fields under intensive corn (maize) production. J Agric Food Chem 24, 122 125

Muir DC, Baker BE (1978) The disappearance and movement of three triazine herbicides and several of their degradation products in soil under field conditions. Weed Res 18, 111-120

Rao PSC, Hornsby AG, Jessup RE (1985) Indices for ranking the potential for pesticide contamination of groundwater. Soil Crop Sci Soc FI Proc $44,1-8$

Ritter WF, Johnson HP, Lovely WG, Molnau M (1974) Atrazine, propachlor, and diazinon residues on small agricultural watersheds. Environ Sci Technol 8, 38-42

Sauer TJ, Daniel TC (1987) Effect of tillage system on runoff losses of surface-applied pesticides. Soil Sci Soc Am J 51, 410-415 
Scheunert I, Qiao Z, Korte F (1986) Comparative studies of the fate of atrazine ${ }^{14} \mathrm{C}$ and pentachlorophenol- ${ }^{14} \mathrm{C}$ in various laboratory and outdoor soil-plant systems. J Environ Sci Health Part B, 21, 457-485

Schiavon M (1980) Contribution à l'étude du mouvement et de la dégradation de l'atrazine dans deux sols agricoles drainés. Interaction matière organique-herbicide. Thèse Dr État Nancy I, $193 \mathrm{p}$

Schiavon M (1988a) Studies of the leaching of atrazine, of its chlorinated derivatives and of hydroxyatrazine from soil using ${ }^{14} \mathrm{C}$ ring-labeled compounds under outdoor conditions. Ecotoxicol Environ Saf 15, 46-54

Schiavon M (1988b) Studies of the movement and the formation of bound residues of atrazine, of its chlorinated derivatives, and of hydroyatrazine in soil using ${ }^{14} \mathrm{C}$ ring-labeled compounds under outdoor conditions. Ecotoxicol Environ Saf 15, 55-61

Schiavon M, Jacquin (1973) Étude de la présence d'atrazine dans les eaux de drainage. In: $C R$ Journ Études Herbicides. Columa, Versailles 1314 décembre 1973, 35-43

Schiavon M, Jacquin F, Goussault C (1978) Blocage de molécules s-triaziniques par la matière organique. IAEA-SM-211/78, 327-332

Schiavon $M$, Thévenot $X$, Andreux F, Portal JM (1990) Relation entre traitements phytosanitaires et contamination des captages. Rapport INPL-Agence de l'Eau-DRASS de Lorraine $72 \mathrm{p}$

Sirons GJ, Frank R, Sawyer T (1973) Residues of atrazine, cyanazine and their phytotoxic metabolites in a clay loam soil. J Agric Food Chem $21,1016-1020$
Snégaroff J (1979) La pollution des eaux par les triazines herbicides dans le secteur du marais charentais. Phytiatr Rev Fr Méd Pharm Vég Phytopharm 28, 249-261

Southwick LM, Willis GH, Bengston RL, Lormand TJ (1990) Atrazine and metolachlor in subsurface drain water in Louisiana. J Irrig Drain Eng $116,16-23$

Triplett GB, Conner BJ, Edwards WM (1978) Transport of atrazine and simazine in runoff from conventional and no-tillage corn. J Environ Qual 7, $77-84$

Von Stryk FG, Bolton EF (1977) Atrazine residues in tile-drain water from corn plots as affected by cropping practices and fertility levels. Can $J$ Soil Sci 57, 249-253

Walker A (1972) The volatility of ${ }^{14} \mathrm{C}$-labelled atrazine and linuron from aluminium planchets. Weed Res 12, 275-278

Wauchope RD (1987) Tilted-bed simulation of erosion and chemical runoff from agricultural fields. II. Effects of formulation and atrazine runoff. $J$ Environ Qual 16, 212-216

Wehtje GR, Spalding RF, Burnside OC, Lowry SR, Leavitt JR (1983) Biological significance and fate of atrazine under aquifer conditions. Weed Sci 31, 610-618

White AW, Barnett AP, Wright BG, Holladay JH (1967) Atrazine losses from fallow land caused by runoff and erosion. Environ Sci Technol 1, 740-744

Wu TL (1980) Dissipation of the herbicides atrazine and alachlor in a Maryland corn field. $J$ Environ Qual 9, 459-465 